\title{
The More, the Better: Is This True in Endoscopy for Gastric Cancer Screening?
}

\author{
Seong Woo Jeon \\ Department of Internal Medicine, School of Medicine, Kyungpook National University, Daegu, Korea
}

See "Factors Affecting Endoscopic Curative Resection of Gastric Cancer in the Population-Based Screening Era" by Yoon Gwon Mun, Myung-Gyu Choi, Chul-Hyun Lim, et al., on page 478-484.

The National Cancer Screening Program (NCSP) in Korea recommends that men and women aged 40 or over should undergo gastroscopy every other year for early detection of gastric cancer. This strategy could be justified by reduced mortality in gastric cancer patients detected by screening endoscopy. Screening endoscopy tends to detect gastric cancer at an early stage, which results in improved survival compared with that in symptomatic patients. A study regarding clinical outcomes of gastric cancer identified by screening endoscopy demonstrated a 5-year survival rate (5-YSR) of $81.3 \%$ in the health-care center in contrast to a 5 -YSR of $53.4 \%$ in the outpatient clinic. ${ }^{1}$ A recent study assessed the efficacy of the Korean NCSP in reducing gastric cancer mortality. Case subjects $(n=54,418)$ were matched with controls $(n=217,672)$ for analysis of survival benefit. The overall odds ratio for death due to gastric cancer among screened subjects was 0.79 (95\% confidence interval, 0.77-0.81). The effect was most prominent in subjects aged 40 to 74 years, and the gastric cancer mortality reduction was $47 \%$ in this age group. ${ }^{2}$

Mun et al. ${ }^{3}$ showed that regular surveillance testing for gastric cancer was an independent factor predicting curative

Received: June 7, 2018 Revised: July 16, 2018

Accepted: July 16, 2018

Correspondence: Seong Woo Jeon

Department of Internal Medicine, School of Medicine, Kyungpook National University Chilgok Hospital, 807 Hoguk-ro, Buk-gu, Daegu 41404, Korea

Tel: +82-53-200-3517, Fax: +82-53-200-2027, E-mail: swjeon@knu.ac.kr ORCID: https://orcid.org/0000-0002-9539-9389

cc This is an Open Access article distributed under the terms of the Creative Commons Attribution Non-Commercial License (http://creativecommons.org/ licenses/by-nc/3.0) which permits unrestricted non-commercial use, distribution, and reproduction in any medium, provided the original work is properly cited. endoscopic resection. Experience and participation in the NCSP were common in curative endoscopic resection group. In other words, patients undergoing gastric cancer screening had a significantly higher rate of endoscopic curative resection, compared with subjects who had never been screened. In addition, the patients who underwent 1-year surveillance screening had a significantly higher rate of endoscopic resection than patients who had no surveillance test in the past 3 years. There was a significant dose-response relationship between surveillance interval and the rate of curative endoscopic resection. These results are similar to that of another Korean study published recently, which showed that triennial endoscopic screening might be as effective as biennial screening in detection of early gastric cancer and subsequent curative endoscopic resection rate. ${ }^{4}$ Another study raised the issue of patients with a high risk of gastric cancer, such as those with intestinal metaplasia, and insisted that screening at 1-year interval would be beneficial in such cases. ${ }^{5}$ However, a recent study analyzing the efficacy of the NCSP showed that mortality reduction was seen even in patients with upper endoscopy interval 48 months or longer. ${ }^{2}$

What is the most appropriated interval for gastric cancer screening by endoscopy? Evidence from published studies showed that currently, biennial or an even longer interval would be beneficial for reducing gastric cancer mortality. However, studies on the benefit of endoscopic resection provide better evidence for shorter screening intervals. Screening is defined as application of diagnostic tests to an asymptomatic person, for the purpose of early intervention. The ultimate purpose of screening is to reduce morbidity and mortality, not 
early detection per se, which would not justify screening programs. ${ }^{6}$ Thus, the measure of efficacy of screening in certain cancers should be based on the reduction of mortality, and this should be achieved in a cost-effective manner. However, more and more studies are aiming for the improvement of quality of life (QOL) after treatment in gastric cancer, which coincide with the development and wide spread use of endoscopic resection for gastric cancer. We can anticipate that the QOL of gastric cancer patients who underwent endoscopic resection would be better than gastrectomized patients. ${ }^{7}$ This paradigm shift from survival benefit to improvement of QOL (in addition to survival) would be the trend in forth-coming studies. The study by Mun et al. is a retrospective study with some limitations such as a lack of QOL data and a potential risk of recall bias because of being questionnaire based. ${ }^{3}$ Also, no data was collected related to the cost-effectiveness of shorter interval. In spite of these limitations, the author has assessed the benefits of screening endoscopy for curability with less invasive treatment. A nation-wide cohort study, including cost-effectiveness and improvement in QOL by endoscopic resection, is warranted to define the appropriate interval of endoscopy for gastric cancer screening.
Conflicts of Interest

The author has no financial conflicts of interest.

\section{REFERENCES}

1. Gong EJ, Ahn JY, Jung HY, et al. Risk factors and clinical outcomes of gastric cancer identified by screening endoscopy: a case-control study. J Gastroenterol Hepatol 2014;29:301-309.

2. Jun JK, Choi KS, Lee HY, et al. Effectiveness of the Korean national cancer screening program in reducing gastric cancer mortality. Gastroenterology 2017;152:1319-1328.e7.

3. Mun YG, Choi MG, Lim CH, et al. Factors affecting endoscopic curative resection of gastric cancer in the population-based screening era. Clin Endosc 2018;51:478-484.

4. Jin S, Jeon SW, Kwon Y, et al. Optimal endoscopic screening interval for early detection of gastric cancer: a single-center study. J Korean Med Sci 2018;33:e166

5. Yoon H, Kim N, Lee HS, et al. Effect of endoscopic screening at 1-year intervals on the clinicopathologic characteristics and treatment of gastric cancer in South Korea. J Gastroenterol Hepatol 2012;27:928-934.

6. Gates TJ. Screening for cancer: evaluating the evidence. Am Fam Physician 2001;63:513-522.

7. Kim SG, Ji SM, Lee NR, et al. Quality of life after endoscopic submucosal dissection for early gastric cancer: a prospective multicenter cohort study. Gut Liver 2017;11:87-92. 